\section{Range-Programming Stripping Voltammetry for Determination of Some Metals in Seawater}

\section{Myung-Zoon Czae and Jong-Hyup Lee}

\author{
Department of Chemistry \\ Hanyang University \\ Seoul 133, Korea
}

Multielement capability of stripping analysis is one of its distinctive advantages described elsewhere [1]. Anodic stripping voltammetry with the use of differential pulse mode (DPASV) is uniquely suited [2], and therefore, applied extensively to the direct simultaneous determination of some trace metals $(\mathrm{Zn}, \mathrm{Cd}, \mathrm{Pb}$, and $\mathrm{Cu})$ in sea waters $[3,4]$. Even with the well-established procedure for a given sample, there still arises the problem introduced by simultaneously measuring relative concentrations of metal ions. A case in point is zinc and cadmium concentrations whose ratio sometimes is as high as 400 (12 ppb $\mathrm{Zn} / 0.03 \mathrm{ppb} \mathrm{Cd}$ ), and typically as high as 200 in raw surface sea waters [3]. In such a case, either one must run the entire steps separately by selecting suitable variables for each metal or pair of metals whose concentration ratio is adequate for simultaneous measurement, or the recorder scale must be changed during a single run to obtain the best results. The latter approach, difficult to achieve manually in practice, would rely on the application of an autoranging amplifier [5]. The use of an autoranging amplifier, however, has given rise to complications in evaluating the resulting voltammograms in most practical applications for analyzing seawaters [6]. The present work was initiated in order to overcome deterioration in readability and data quality, by developing a new programmedranging technique.

\section{Principle of Operation}

With the pulsed (commonly DP) stripping mode in the simultaneous measurements, each voltammogram peak starts from the baseline of nearly zero or very small current when moderate resolution is provided. This fact enables us to change current gain to a pre-specified value (programmed vs potential, according to the concentration ratios for a lot of samples), when the potential is scanned to a position just before the start of a subsequent peak (where pre-peak current also drops nearly to the baseline). Figure 1 illustrates the basic principle behind the programmed-ranging technique. If we incorporate an additional amplifier (fig. 1, lower large box) with a gain (programmable) of $G_{j}(x)$ to a prototyping polarographic analyzer's output (which otherwise would give the peak current $i_{j}$, for the $j$ th component or peak), the overall analytical signal $\left(I_{j}\right)$ will be

$$
I_{j}=G_{j}(x) i_{j}
$$

where gain $G_{j}(x)$ depends on some variable $x$. In a scheme employing an autoranging amplifier, $x$ is $I_{j}$ provided by feedback means. Thus, gain $G_{j}\left(I_{j}\right)$ varies indefinitely in a stepwise manner by a predetermined factor of $(10)^{1 / 2}, 10$, or several decades. This approach requires the operator's attention for marking the gain increments. We adopted a potential window, $E_{\langle j\rangle}$, as $x$, located around the $j$ 's peak potential. The implementation of eq (1) with $G_{j}\left(E_{\langle j}\right)$, range programming (vs potential), can be provided by feedforward automatically, instead of by feedback.

The gain or range programming amplifier unit (fig. 1) comprises a comparator with variable threshold (Reference Voltage) and associated circuitry (Processing Ckt) for automatically changing the gain $G_{j}\left(E_{(j)}\right)$ when the scanned electrode potential reaches a threshold of the $j$ th potential window $E_{\langle j\rangle}$. The value of gain $G_{j}$ remains at the programmed value over the entire potential window $E_{(j)}$. The processing circuit comprises a programmable gain amplifier, counter, and multiplexer/demultiplexer (MPX) and related circuitry for performing the proper functions. By comparing the sweep potential with the set voltage, the comparator causes the counter to produce a bit control signal to the demultiplexer, which selects a gain-determining feedback resistor, and, in turn, switches the next prespecified reference voltage to the comparator.

\section{Results and Discussion}

Figure 2 shows differential pulse stripping voltammograms with a range or gain program shown in (C) for a $10 \mathrm{~mL}$ aliquot of the filtered seawater sample solution $(0.45 \mu \mathrm{m}$ Millipore 
membrane) whose pH was adjusted to 3.0. The adverse concentration ratio $(\mathrm{Zn} / \mathrm{Cd})$ was 150 . The readability, however, improved to the same order of magnitude in the range programming mode ((b) in fig. 2), without making any sacrifice in data quality. The potentiostat was a PAR Model 174A equipped with a Model 303 static mercury drop electrode.

To establish linearity between peak current and concentration, standard curves for four metals in seawater were simultaneously run at $\mathrm{pH}$ 3.0. Standard additions were made by adding to the $10-\mathrm{mL}$ sample, $10-\mu \mathrm{L}$ volumes of a solution containing $0.100 \mathrm{ppm}$ in $\mathrm{Cd}$ and $1.0 \mathrm{ppm}$ in $\mathrm{Zn}, \mathrm{Pb}$, and $\mathrm{Cu}$. The resulting standard addition curves (shown in fig. 3) demonstrate response linearity with good precision. With the deposition potential of $-1.2 \mathrm{~V}$ (vs $\mathrm{Ag} / \mathrm{AgCl}$ ) and a deposition time of $3 \mathrm{~min}$, we applied the technique to analyze simultaneously more than 300 seawater and marine samples employing the standard addition calibration curve and triple spiking procedure [7], respectively.

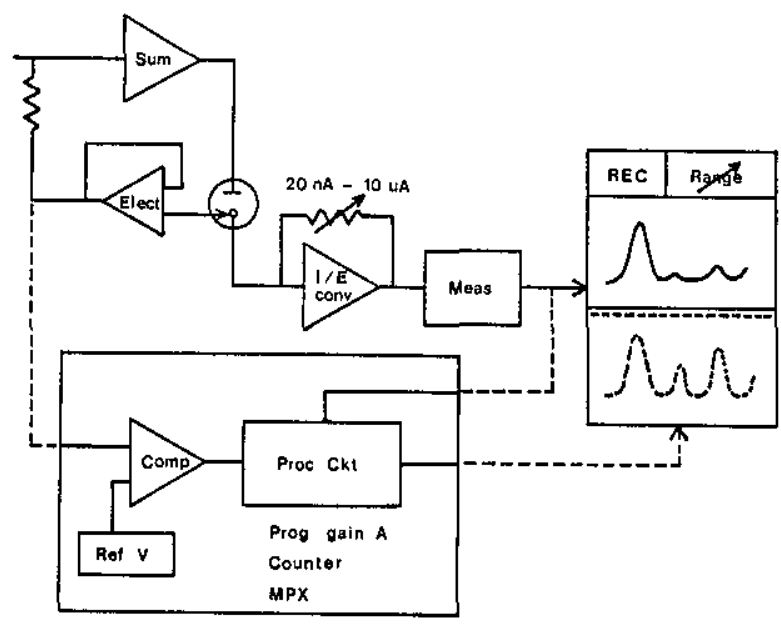

Figure 1. Schematic diagram of proposed technique.

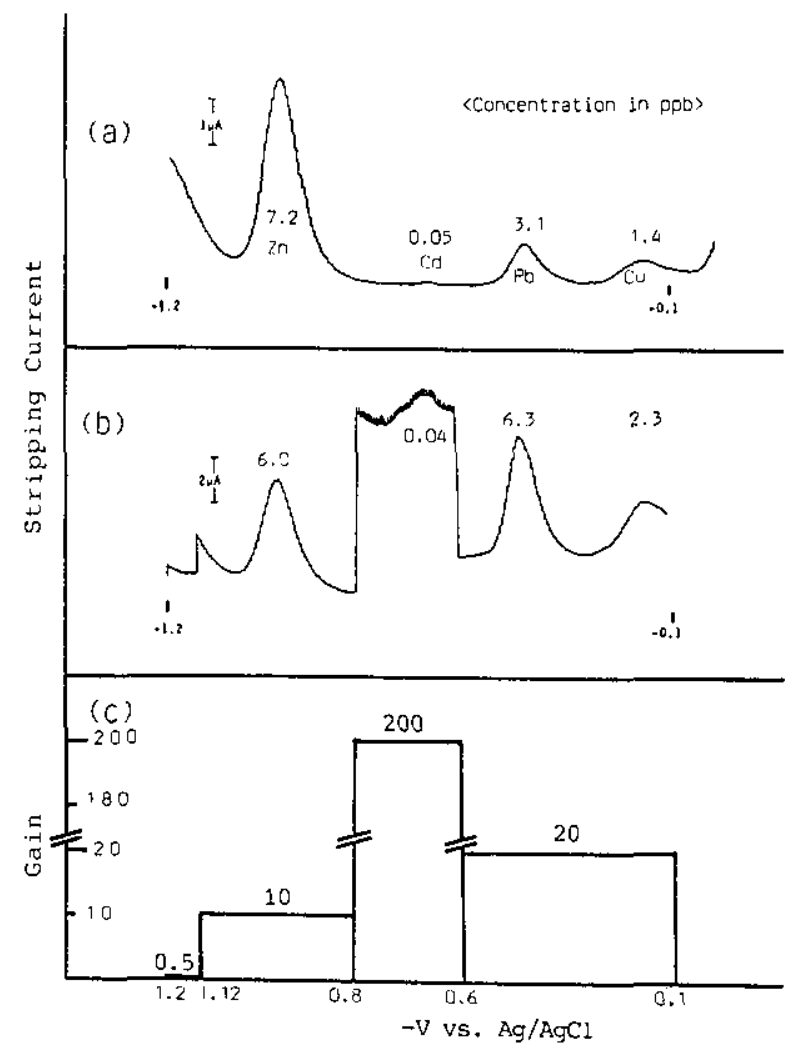

Figure 2. Typical voltammograms, (a) conventional, with deposition time of $30 \mathrm{~min}$; (b) range programmed, with $3 \mathrm{~min}$ deposition with gain program shown in (c).

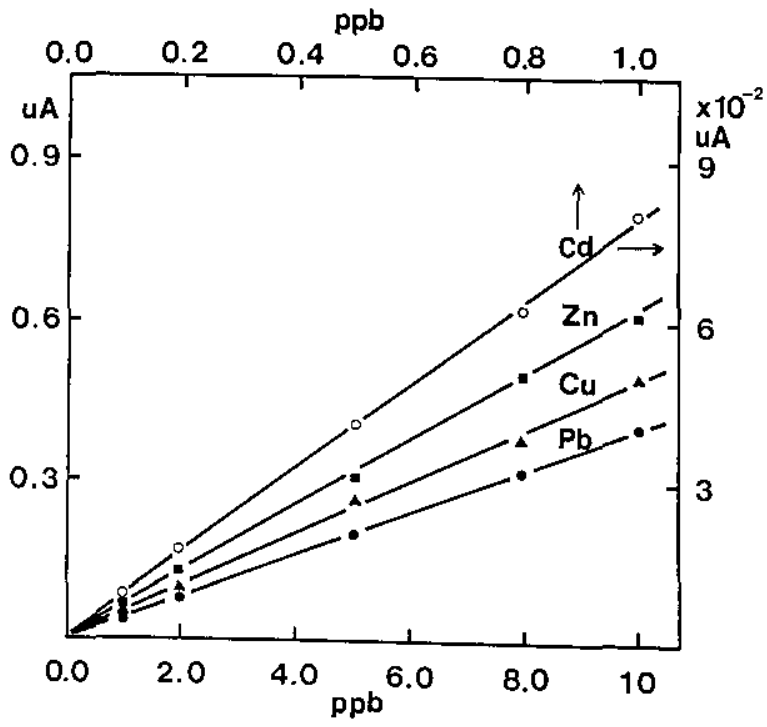

Figure 3. Standard addition calibrating curves in seawater. The peak currents are net values. 


\section{References}

[1] Wang, J., Envir. Sci. Technol. 16, 104A (1982); Stripping Analysis, VCH Publishers, Inc., Deerfield Beach, FL (1985) Chap. 1.

[2] Zirino, A., Voltammetry of Natural Sea Water, in Marine Electrochemistry, M. Whitfield and D. Jagner, eds., John Wiley and Sons, New York (1981) Chap. 10.

[3] Czae, M.-Z., and Lee, J.-H., Bull. Envir. Sci. (Hanyang Univ., Seoul, Korea) 6, 153 (1985).

[4] Kim, E., Czae, M.-Z., et al., Studies on the Effect of Cooling Water to the Cultivated Marine Organism around Poryung and Samchunpo Power Plants, Part I and II, KRC-84C-J01, Korea Electric Corp., Seoul, March 1985.

[5] Ben-Yaakov, S., and Lazar, B., Talanta 27, 1061 (1980).

[6] Lee, J.-H., Autoranging Amplifier for the Simultaneous Determination of Some Trace Metals in Seawater by DPASV. Thesis (MS), Hanyang University, Seoul, Korea.

[7] Czae, M.-Z., Lee, J.-H., and Hong, T., Validation of Anodic Stripping Voltammetry for Determination of Some Metals in Sea Samples, Proc. First Korea-Japan Joint Symp. on Anal. Chem. (Korean Chem. Soc.), Seoul, Korea (April 1985) 27-36.

\section{Computer Assisted Pesticide and PCB Identification System (CAPPIS)}

\author{
Joseph E. Dierkes \\ New Jersey State Department of Health \\ John Fitch Plaza CN 361 \\ Trenton, NJ 08625
}

Environmental samples (water, air, soil, etc.) are routinely analyzed in the state laboratory for pesticide and PCB residues using gas chromatographs (GCs) equipped with electron capture detectors (ECDs), and packed glass $(4 \mathrm{~mm} \times 2 \mathrm{~m})$ columns of differing polarity. In a typical analysis, one primary column (1.5\% OV-17/1.95\% OV-210) is used to quantitate results obtained after confirmation by a secondary column (4\% SE-30/6\% OV-210) and occasionally a third column (5\% OV-210, 3\% OV1, etc.).

Two standard mixtures of pesticides are injected before any samples are analyzed. After verifying retention times (RTs) for key pesticides (i.e., pp'. DDT) in these standards, actual sample analysis may begin. Should any peaks be encountered, then their RTs are compared with those of the standards. If the sample peak RT on each column used matches the RT of the pesticide standard on each column within certain limits, identification is tentatively confirmed. If a multicomponent residue such as chlordane, toxaphene, or a PCB is found, then the appropriate or suspected standard is also injected and chromatographed. Each sample chromatogram is checked for the presence and proper RT of any surrogate compounds (Mirex or dibutylchlorendate) used.

The peak height ratios between the columns are next examined for a similar ratio obtained for that of the pesticide and the standard, thus ruling out false identifications due to interferences. Following U.S.E.P.A. practice, positive matches are quantitated by a comparison (peak height or area) with known concentrations of standards, as long as the peak heights between sample and standards are within $25 \%$ of each other. If the positive result is the surrogate compound, then its percent recovery is also calculated.

It was desired to develop a computer algorithm to perform all these checks and measurements with greater accuracy and speed, provide a considerable amount of quality control documentation for large numbers of samples, and at the same time meet or surpass stringent U.S.E.P.A. and IFB analytical requirements.

To this end, a battery of GCs was interfaced with a mainframe computer system. Sample chromatograms are produced by standard $1 \mathrm{mV}$ recorders and the GC signals are simultaneously digitized by analog-to-digital converters. The digitized data from each analysis are arranged into a definite set of records in a file by a modified post run program which assigns the file a unique number and stores it on the disk. Any chromatogram can be called up at a later time for access by the software package, designated CAPPIS, by referring to its file number.

This package consists of a set of simple BASIC/ FORTRAN programs which could be modified to work with various analytical systems. All that would be needed would be to set up unique digitized data files and define the ways the software could access them.

After a GC is set up, its operating parameters (column types, carrier gas type and flow rate, temperature, etc.) are stored on the disk in a file generated by one of these programs. Next, retention time windows are generated by another program and also stored on disk. This completes the initial setup, and is essentially done once. Any later changes in the operating parameters, columns, or windows are 